\title{
Analysis of Forces during Robotic Needle Insertion to Human Vertebra
}

\author{
Kiyoshi Matsumiya ${ }^{1}$, Yasuyuki Momoi ${ }^{2}$, Etsuko Kobayashi ${ }^{3}$, \\ Nobuhiko Sugano ${ }^{4}$, Kazuo Yonenobu ${ }^{5}$, Hiroshi Inada ${ }^{1}$, \\ Takayuki Tsuji ${ }^{3}$, and Ichiro Sakuma ${ }^{3}$ \\ ${ }^{1}$ Graduate School of Engineering, The University of Tokyo \\ ${ }^{2}$ Hitachi Ltd. \\ ${ }^{3}$ Graduate School of Frontier Sciences, The University of Tokyo \\ ${ }^{4}$ Graduate School of Medicine, Osaka University \\ ${ }^{5}$ Osaka Minami National Hospital \\ mkiyo@miki.pe.u-tokyo.ac.jp
}

\begin{abstract}
The purpose of this study is assessment of forces and torques required for robotic needle insertion to human vertebra. Axial forces during robotic insertion to human vertebrae fixed with formalin (water solution of formaldehyde(FA)) did not exceed $25 \mathrm{~N}$ when the feed rate was no more than 0.5 $\mathrm{mm} / \mathrm{s}$. There was relatively strong correlation between the axial force variation during insertion and the distribution of bone local CT-value along the needle path. This result shows the importance of preoperative X-ray CT image guidance for robotic needle insertion with safely small axial forces. There was no significant influence of FA-fixation on axial forces during robotic needle insertion to human femoral heads. The forces during robotic insertion to human femoral head were as small as less than $50 \%$ of those during manual insertion, and which indicates that the robot can contribute to the safe needle insertion to human vertebra in percutaneous vertebroplasty.
\end{abstract}

Keywords: Percutaneous vertebroplasty, needle insertion, robot, forces and torques, CT-value.

\section{Introduction}

Percutaneous vertebroplasty is the percutaneous injection of prosthetic material such as bone cement to stabilize and strengthen a collapsing vertebral body, and is one of the minimal invasive therapies for osteoporotic compression fracture of vertebra. In the procedure, a needle is inserted to the vertebra under guidance of X-ray fluoroscopy, C-arm. Conventionally, the surgeon performs this process manually, and therefore there are two major challenges as follows: (1) Surgeon is requested to have excellent skill in needle insertion without direct view. (2) Surgeon must take a risk of Xray exposure to his or her hands during the procedure.

One promising method to relieve these challenges is the introduction of robotic needle insertion. Though there is no report on robotic needle insertion to the bone in clinical use, robots have been successfully used in accurate milling of the bone such as in total knee replacement (TKR)[1,2]. On the other hand there is a report of manual insertion using needle holder for reduction of X-ray exposure to surgeon[3], and ro- 
bots contributed similarly to the holder. There are many reports on surgical robots using X-ray imagers, in which the surgeon can avoid excessive X-ray exposure[4-7].

The purpose of this study is assessment of forces and torques required for robotic needle insertion to human vertebra for development of robotic assistance of this procedure.
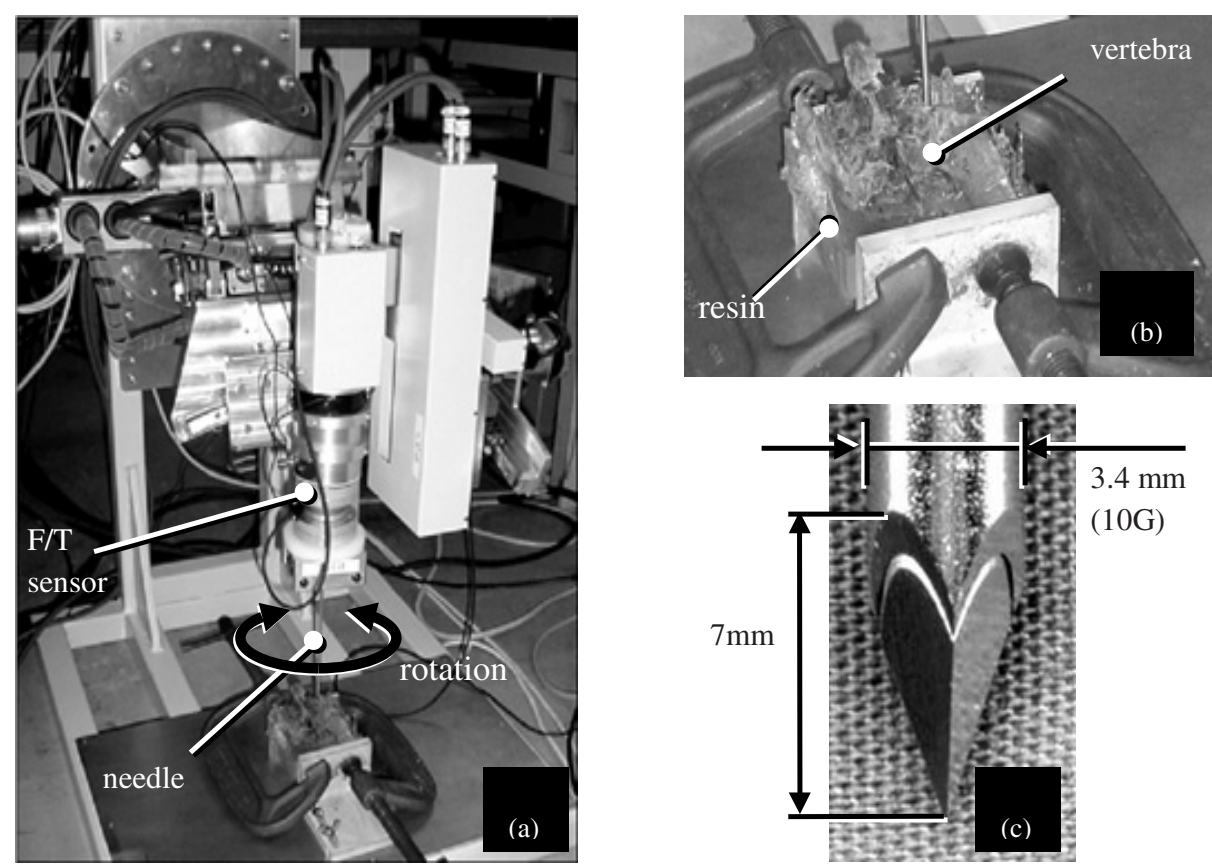

Fig. 1. The robot (a) inserted the needle with bi-directonal rotation around the axis to the vertebra embedded in resin and fastened with a vise (b). The needle (c) was about $3.4 \mathrm{~mm}$ in diameter and attached to the robot via a force/torque sensor.

\section{Materials and Methods}

\subsection{Measurement of Forces and Torques during Needle Insertion to FA-Fixed Human Vertebrae}

Robotic needle insertion into human cadaveric vertebra was performed measuring forces that developed between the needle and vertebra. All the vertebrae had been preserved under formalin (water solution of formaldehyde(FA)) fixation, were embedded in resin that had been hardened in a mold, and then were well fastened with a vise on the base (Fig.1.b). A commercially used needle (Stryker, USA) (Fig.1.c) of $3.4 \mathrm{~mm}$ diameter (10 gauge) was attached to the robot (Fig.1.a) via a force/torque 
sensor (MINI 8/40, BL-Autotech, Japan) connected to a data processing PC. The robot, which had been newly developed for percutaneous vertebroplasty, makes it possible to insert the needle with bi-directional rotation avoiding involution of blood vessel, nerve and other tissues.

Before each insertion, the entry point and orientation of the needle were planned manually under direct view by the investigators. The needle was inserted into the vertebral body up to $30 \mathrm{~mm}$ depth from the initial point straightly through the pedicle. The bi-directional rotation angle was \pm 80 degree (Fig. 2). A protocol was chosen for each insertion among the sets of feed rate and rotation speed (Table 1). The forces and torques were measured continuously with force/torque sensor from the start to the end of each insertion.

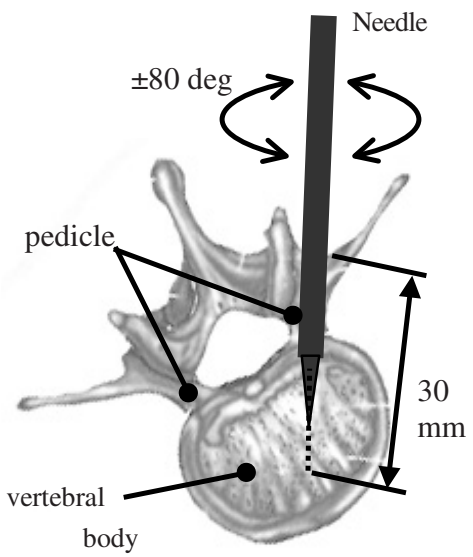

Fig. 2. The needle was inserted up to $30 \mathrm{~mm}$ depth into vertebral body through pedicle. The rotation angle was $\pm 80 \mathrm{deg}$.
Table 1. Protocol of robotic needle insertion to FA-fixed human vertebra.

\begin{tabular}{r|c|c|c|c|c}
\hline \hline Protocol \# & 1 & 2 & 3 & 4 \\
\hline Rotation angle [deg] & \multicolumn{5}{|c}{ \pm 80} \\
\hline Feed rate [mm/s] & 0.05 & 0.05 & 0.2 & 0.5 \\
\hline $\begin{array}{r}\text { Rotaion speed [deg/s] } \\
([\mathrm{Hz}])\end{array}$ & 840 & 600 & 840 & 840 \\
$(2.63)$ & $(1.88)$ & $(2.63)$ & $(2.63)$ \\
\hline
\end{tabular}

Table 2. Protocol of robotic needle insertion to human femoral

\begin{tabular}{r|c|c|c}
\hline \hline Protocol \# & 1 & 2 & 3 \\
\hline Rotation angle [deg] & \multicolumn{3}{|c}{ \pm 80} \\
\hline Feed rate [mm/s] & 0.2 & 0.2 & 1.0 \\
\hline Rotaion speed [deg/s] & 840 & 600 & 840 \\
$([\mathrm{~Hz}])$ & $(2.63)$ & $(1.88)$ & $(2.63)$ \\
\hline
\end{tabular}

\subsection{Analysis of Axial Forces and CT-Values}

After the experiment described in 2.1, all the vertebrae were scanned with X-ray CT (slice interval: $3 \mathrm{~mm}$, field of view (FOV): $250 \mathrm{~mm}$ in diameter, 512 x 512 pixels, 2 byte/pixel). The path of the needle insertion was confirmed on the cross sectional images. On assumption that CT-value acquired from X-ray CT image of the bone is proportional to the bone mineral density (BMD) and thus the mechanical strength of the bone, the relationship between axial forces and CT-values was analyzed as follows: First of all, the cross sections clearly including the needle path were chosen for the analysis. In each cross sectional images, the area of the bone near the needle path was divided into small boxes of 9 pixels ( 3 rows of 3 pixels), and the average CT-values were calculated in all boxes. The CT-values along the needle path were calculated as described in Fig. 3. 


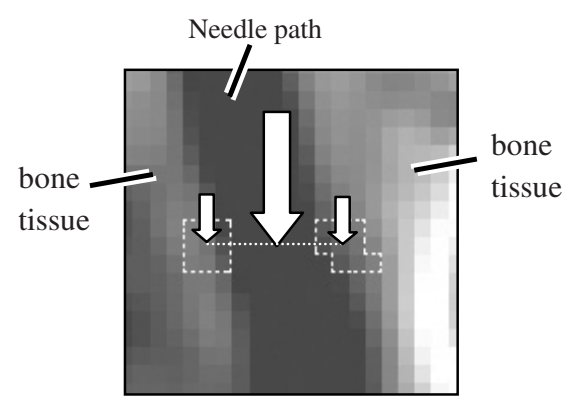

Fig. 3. Each box consists of 9 pixels. After calculating average CT-value of 2 paring boxes (small pointers), average CT-value of them is calculated as the CT-value along the needle path (large pointer).

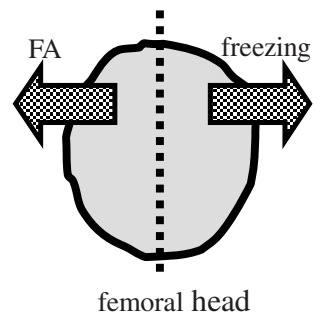

Fig. 4. Fresh femoral head was divided into 2 parts; one was for FAfixation, the other was for freezing.

\subsection{Investigation into the Influence of FA-Fixation on the Axial Force}

It is possible that FA-fixation changes mechanical stiffness of the bone. In order to investigate the influence of FA-fixation on the axial force, axial forces during insertion to the human femoral heads that had been preserved under freezing or FAfixation were measured and compared each other. It was assumed here that there was no significant difference in mechanical properties between fresh and defrosted bones. All the test pieces were obtained in the procedure that each fresh femoral head was divided into two parts; one was for FA-fixation and the other was for freezing (Fig. 4.): It was assumed that both parts of a pair had similar mechanical property before preservations by 2 different means, and which helps to reduce the weight of difference in mechanical property of the bone on the data dispersion. Each test piece was fastened with a vise on the base. The needle was inserted up to $12 \mathrm{~mm}$ depth under a set of the feed rate and rotation speed shown in Table 2. The other materials and protocols were the same as in the experiment described in 2.1 .

\subsection{Comparison between Manual and Robotic Insertion}

Manual needle insertion into defrosted and FA-fixed human femoral heads was done. The needle was inserted manually by a well-trained orthopedic surgeon into the same test pieces that had been used in robotic insertions; each test piece was inserted both robotically and manually and which helps reduction of data dispersion as well as mentioned in 2.3.

The needle was attached to a handy device with the force/torque sensor (Fig. 5.). Optical markers were also attached on this device for the measurement of 3D position of the needle using the optical sensor system Optotrak (Northern Digital, USA). Needle was inserted up to depth of more than $12 \mathrm{~mm}$. The other materials and the protocols were the same as previous experiments. 


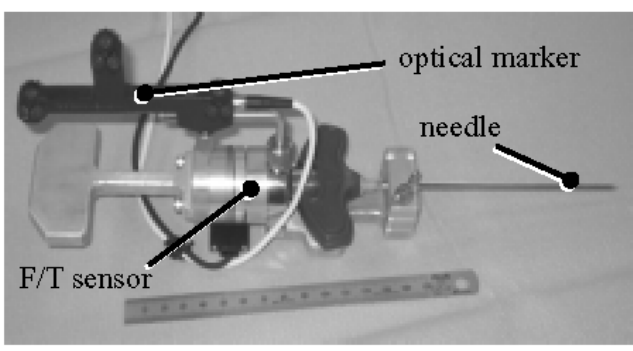

Fig. 5. The device for manual neelde insertion with attached $\mathrm{F} / \mathrm{T}$ sensor and opical markers.

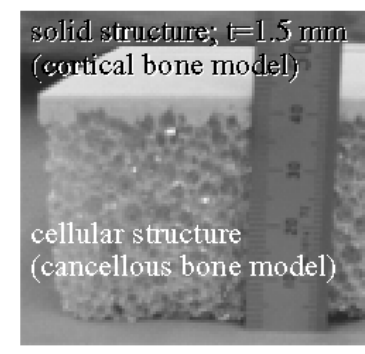

Fig. 6. Polyurethane phantom Sawbones für preliminary tests.

\section{Result and Discussion}

\subsection{Forces and Torques during Needle Insertion to FA-Fixed Human Vertebrae}

In general, the thickness of cortical bone near the pedicle, through which the needle was inserted, is approximately $1-2 \mathrm{~mm}$. The length of edged part of a needle near the tip was approximately $7 \mathrm{~mm}$ (Fig. 1.c). Under these conditions, it was assumed that the axial force would be at the maximum usually when the needle tip was at the depth of $0-10 \mathrm{~mm}(\mathrm{D}=0-10 \mathrm{~mm})$. This had been suggested by the result of a preliminary experiment using polyurethane phantom Sawbones (Pacific Research Laboratories, USA) (Fig. 6.): The forces were at the maximum when $\mathrm{D}=4.02 \mathrm{~mm}$ (S.D. \pm 0.61 $\mathrm{mm}, \mathrm{n}=15$ ), which was absolutely included in the section of $\mathrm{D}=0-10 \mathrm{~mm}$. However, in this experiment using human vertebrae, axial forces were at the maximum in the section of $\mathrm{D}=0-10 \mathrm{~mm}$ only in 11 insertions of the total 23 insertions. The depth where the axial force was at the maximum was D $=13.11 \mathrm{~mm}$ (S.D. $\pm 8.93 \mathrm{~mm}, \mathrm{n}=$ 23).

Axial forces did not exceed $25 \mathrm{~N}$ in all insertions. The maximum axial force was large in insertions at large feed rate (Fig. 7. left), which is consistent with a report on the robotic biopsy needle insertion to bone-like phantom[8]. The maximum torque was slightly large in insertions at large feed rate or at small rotational speed (Fig. 7. right), probably because of the large friction moment of the bone during one rotation of the needle.
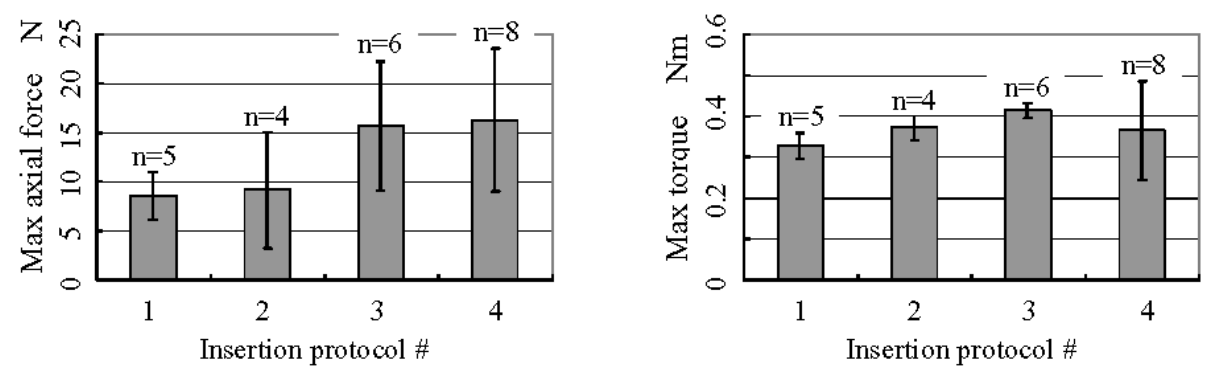

Fig. 7. Max force (left) and torque (right) during the insertion to vertebra (mean \pm S.D.). 


\subsection{Analysis of Axial Forces and CT-Values}

The examples of axial force variation during the insertion and the corresponding cross sectional X-ray CT images suggest that axial force depended on the local stiffness of the bone (Fig. 8). They also suggest that axial force would be comparably small beyond $\mathrm{D}=10 \mathrm{~mm}$ if the insertion path properly escaped from the hard part of vertebra displayed brightly in X-ray CT images.

In this analysis, 15 paths were the targets among totally 23 paths. Each of these 15 paths was clearly displayed on one cross sectional image, and which was appropriate for the accurate calculation of CT-values avoiding the problem such as partial volume. The correlation coefficient regarding the axial force and CT-value was 0.35 (S.D. \pm $0.24, \mathrm{n}=15)$; there was relatively strong correlation between the axial force variation during insertion and the distribution of bone local CT-value along the needle path. This result shows the importance of preoperative X-ray CT image guidance for robotic needle insertion with safely small axial forces. Figure 9 indicates a typical case in which there was strong correlation between the axial force and CT-value (coefficient $=0.82$ ).
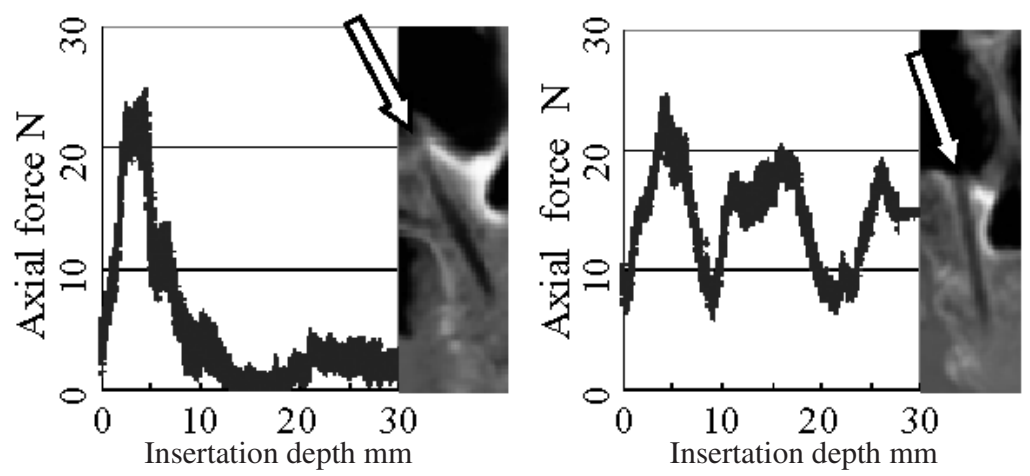

Fig. 8. Examples of axial force changes and the corresponding cross sectional X-ray images. The pointer indicates entry point.
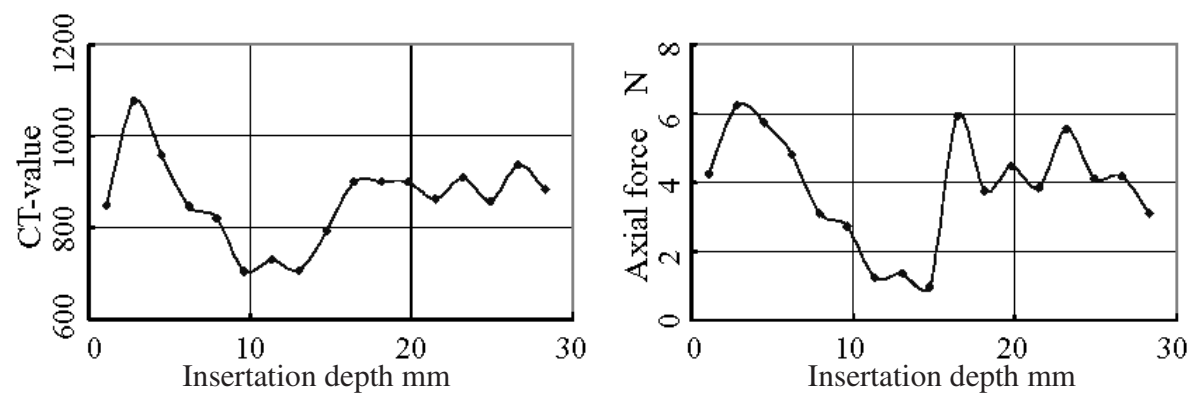

Fig. 9. A typical case of strong correlation between axial force (right) and CT-values (left). Correlation coefficient $=0.82$. 


\subsection{Investigation into the Influence of FA-Fixation on the Axial Force}

Currey et al reported that FA-fixation increased the stiffness itself of a bone but decreased the energy required for breaking a bone [9], and their result suggested that FA-fixation maybe make it easy to break a bone. The result in this experiment did not necessarily follow their report. Figure 10 indicates that there was no significant difference between bones preserved under FA-fixation and freezing. Further investigation about the influence of FA-fixation on the mechanical property might be required.

\subsection{Comparison between Manual Insertion and Robotic Insertion}

Much smaller values of the maximum axial force were measured during robotic insertion than manual insertion (Fig. 11.). The ratio of the maximum axial force (robot/manual), each of which was calculated among 2 insertions to the same test piece, was as small as $34.0 \%$ (S.D. $\pm 12.1 \%, \mathrm{n}=8$ ) using FA-fixed bones and $28.3 \%$ (S.D. $\pm 16.8 \%, \mathrm{n}=9$ ) using defrosted bones. These results suggest that the robot realized the reduction of axial force, which helps safer needle insertion in percutaneous vertebroplasty. The robot can hold the needle more stably during insertion than human. Moreover it can rotate the needle with larger rotation angle at larger rotation speed than human. These factors might be the reasons why robotic insertion required smaller axial forces than manual insertion.

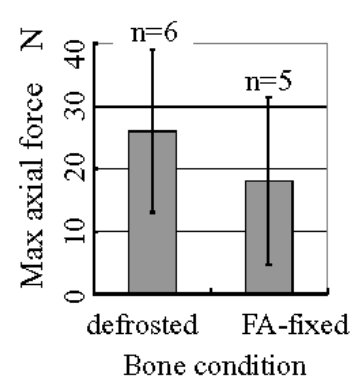

Fig. 10. Max axial force during insertation to femoral head under 2 conditions (mean \pm S.D.).
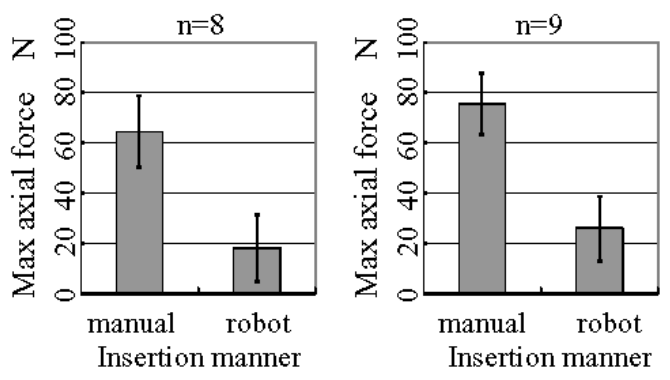

Fig. 11. Max axial force during insertation to FA-fixed (öeft) and defrosted (right) femoral head manually und robotically (mean \pm S.D.).

\section{Conclusion}

Forces and torques during robotic insertion to FA-fixed human vertebrae were measured and the forces did not exceed $25 \mathrm{~N}$ in all cases when the feed rate was $0.05-0.5$ $\mathrm{mm} / \mathrm{s}$. There was relatively strong correlation between the axial force variation during insertion and the distribution of bone local CT-value along the needle path, which is promising for the appropriate needle path planning based on the preoperative $\mathrm{X}$-ray CT images. There was no significant influence of FA-fixation on axial forces during robotic needle insertion to human femoral heads. The forces during robotic insertion to human femoral head were much maller than those during manual insertion, and which indicates that the robot can contribute to the safe needle insertion to human vertebra in percutaneous vertebroplasty. 
Acknowledgements. This study is partly supported by JSPS-RTFT99I00904.

\section{References}

1. M. Jakopec, S. J. Harris, F. Rodriguez y Baena, P. Gomes, J. Cobb, and B. L. Davies, The first clinical application of a "hand-on" robotic knee surgery system, Computer Aided Surgery 2001; 6: 329-339.

2. W. Siebert, S. Mai, R. Kober, and P. F. Heeckt, Technique and first clinical results of robotassisted total knee replacement, Knee 2002; 9: 173-180.

3. R. Kato, K. Katada, H. Anno, S. Suzuki, Y. Ida, and S. Koga, Radiation dosimetry at CT fluoroscopy: physician's hand dose and development of needle holders, Radiology 1996; 201: 576-578.

4. J. A. Cadeddu, A. Bzostek, S. Schreiner, A. C. Barnes, W. W. Roberts, J. Anderson, R. H. Taylor, and L. R. Kavoussi, A robotic system for percutaneous renal access, Journal of Urology 1997; 158: 1589-1593.

5. K. Cleary, M. Freedman, M.Clifford, D. Lindisch, S. Onda, and L. Jiang, Image-guide robotic delivery system for precise placement of therapeutic agents, Journal of Controlled Release 2001; 74: 363-368.

6. K. Masamune, G. Fichtinger, A. Patriciu, R. C. Susil, R. H. Taylor, L. R. Kavoussi, J. Anderson, I. Sakuma, T. Dohi, and D. Stoianovici, System for robotically assisted percutaneous procedures with computer tomography guidance, Computer Aided Surgery 2001; 6: 370-383.

7. S. B. Solomon, A. Patriciu, M. E. Bohlman, L. R. Kavoussi, and D. Stoianovici, Robotically driven interventions: a method of using CT fluoroscopy without radiation exposure to physician, Radiology 2002; 225: 277-282.

8. A. D. Heiner, T. D. Brown, V. Rossin, and J. A. Buckwalter, Frictional insertion kinetics of bone biopsy needles, Journal of Biomechanical Engineering 2001; 123: 629-634.

9. J. D. Currey, K. Brear, P. Zioupos and G. C. Reilly, Effect of formaldehyde fixation on some mechanical properties of bovine bone, Biomaterials 1995; 16: 1267-1271 